\title{
Saccular Pathology Is Most Commonly Found in Patients With General Vestibular Disorders
}

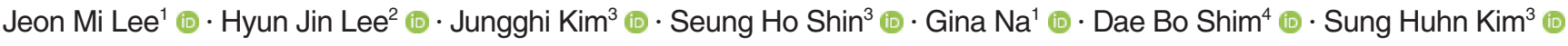 \\ ${ }^{1}$ Department of Otorhinolaryngology, Ilsan Paik Hospital, Inje University College of Medicine, Goyang; \\ ${ }^{2}$ Department of Otorhinolaryngology, Incheon St. Mary's Hospital, College of Medicine, The Catholic University of Korea, Seoul; \\ ${ }^{3}$ Department of Otorhinolaryngology, Yonsei University College of Medicine, Seoul; \\ ${ }^{4}$ Department of Otorhinolaryngology, Myongji Hospital, Hanyang University College of Medicine, Goyang, Korea
}

Objectives. The first purpose of this study was to investigate the difference in the frequency of involvement of the superior vestibular nerve (SVN) and inferior vestibular nerve (IVN) territories in general vestibular disorders, and to identify which IVN territory was more commonly involved in patients with IVN lesions. The second purpose was to investigate the correlation of the degree of each saccular and posterior semicircular canal (PSCC) dysfunction, as represented by the parameters of cervical vestibular evoked myogenic potential (cVEMP) and video head impulse test (vHIT), in patients with pathology of the IVN territory.

Methods. In total, 346 patients with dizziness who underwent the caloric test, cVEMP, and vHIT were enrolled. Canal weakness in the caloric test, interaural amplitude difference (IAD) of cVEMP, and vestibulo-ocular reflex gain of the vestibulo-ocular reflex gain of the posterior semicircular canal (p-VOR) in vHIT were analyzed.

Results. Among the enrolled patients, $15.6 \%$ had total vestibular nerve dysfunction, $14.5 \%$ had solely SVN dysfunction, and $29.5 \%$ had solely IVN dysfunction. Isolated saccular pathology was most common in patients with IVN pathology, followed by those with total IVN dysfunction and PSCC dysfunction. IAD and p-VOR were statistically well correlated, and the correlation was strongest in patients with both pathologic IAD and pathologic $\mathrm{p}-\mathrm{VOR}(\mathrm{n}=23$, $r=0.944)$, followed by patients with normal IAD and pathologic $\mathrm{p}$-VOR $(\mathrm{n}=27, r=0.762)$ and patients with pathologic IAD and normal $\mathrm{p}$-VOR $(\mathrm{n}=106, r=0.339)$.

Conclusion. Abnormal results were more common in vestibular tests investigating the IVN than in vestibular tests investigating the SVN in patients with general vestibular disorders. Isolated saccular pathology was more frequent than PSCC or combined pathology in patients with IVN dysfunction. Patients with abnormal p-VOR in vHIT had a higher probability of having both saccular and PSCC pathologies than patients with an abnormal IAD. This study describes the characteristics of vestibular-system subregions and provides guidance for clinically interpreting the combination of cVEMP and vHIT results.

Keywords. Head Impulse Test; Vestibular Evoked Myogenic Potentials; Vestibular Nerve; Dizziness

\section{INTRODUCTION}

- Received April 26, 2021

Revised September 9, 2021

Accepted September 18, 2021

- Corresponding author: Sung Huhn Kim

Department of Otorhinolaryngology, Yonsei University College of Medicine,

Severance Hospital, Yonsei University Health System, 50 Yonsei-ro,

Seodaemun-gu, Seoul 03722, Korea

Tel: +82-2-2228-3620, Fax: +82-2-393-0580

E-mail: fledermaus@yuhs.ac
The vestibular system, which is responsible for maintaining balance, comprises three semicircular canals, the ampulla, the utricle, and the saccule. The superior and horizontal semicircular canals and utricle are innervated by the superior vestibular nerve (SVN), and the posterior semicircular canal (PSCC) and saccule are mostly innervated by the inferior vestibular nerve (IVN) [1]. Although most dizziness and imbalance symptoms are caused

Copyright () 2022 by Korean Society of Otorhinolaryngology-Head and Neck Surgery.

This is an open-access article distributed under the terms of the Creative Commons Attribution Non-Commercial License (https://creativecommons.org/licenses/by-nc/4.0)

which permits unrestricted non-commercial use, distribution, and reproduction in any medium, provided the original work is properly cited. 
by pathological changes in the vestibular system, it is often difficult to find the precise pathological region of each compartment of the system using only neuro-otological examinations with the naked eye. For this reason, remarkable developments have been made in test approaches for dizziness and imbalance over the past few years. The most noteworthy approaches recently developed for the evaluation of vestibular function are the cervical and ocular vestibular evoked myogenic potential (cVEMP and oVEMP) testing and the video head impulse test (vHIT), respectively. These assessments enable neuro-otologists to assess the function of each vestibular end organ objectively and quantitatively, providing a basis for physicians and researchers to differentiate the pathological subregion of the vestibular system. With the help of novel test approaches, it has been reported that lesions in the SVN territory are more common in patients with acute vestibulopathy than those in the IVN territory in patients with acute vestibulopathy [2]. Although the symptoms of vestibulopathy in the territory of the IVN are not as severe as those in the territory of the SVN, it can also cause acute and chronic dizziness and imbalance; therefore, interest in pathologies in the territory of the IVN is increasing.

cVEMP evaluates the integrity of saccular function by evaluating the sacculocolic reflex induced by sound or vibration stimulation. The results of cVEMP represent the function of the saccule and the IVN innervation of the saccule. The vHIT measures the vestibulo-ocular reflex in the three semicircular canals. In vHIT, the results of the test for the PSCC represent the function of the PSCC innervated by the IVN. If a pathology is confined to the PSCC or the saccule, only one of the two tests would show an abnormal finding, but if a pathology initiates from a more proximal portion of the IVN, both tests would show abnormal findings. Many studies have examined whether patients with unilateral vestibular hypofunction have combined pathologies in both the saccule and PSCC or isolated pathologies [3-5]; however, these studies were conducted with selected patients

\section{H I G H L I I G H T S}

- Abnormal results are more frequently found in vestibular tests investigating the inferior vestibular nerve (IVN) than in vestibular tests investigating the superior vestibular nerve in patients with general vestibular disorders.

- Isolated peripheral end organs, particularly saccules, are likely to be more vulnerable than the proximal IVN in patients with IVN dysfunction.

- Patients with pathologic vestibulo-ocular reflex gain of the posterior semicircular canal had a higher likelihood of having both saccular and posterior semicircular canal pathologies than patients with a pathologic interaural amplitude difference.

- The combination of cVEMP and vHIT could elucidate dizzy patients' clinical pathway. with acute unilateral vestibulopathy, and no studies have investigated the correlation between cVEMP and vHIT in a large population with general vestibular disorders.

Therefore, in this study, we investigated the distribution of vestibular lesion sites (SVN or IVN) in a large population of patients who visited a hospital due to dizziness. We further investigated the differences in the frequency of total IVN dysfunction, isolated saccular dysfunction, and isolated PSCC dysfunction by comparing the results of cVEMP with PSCC function in vHIT to determine which pathology is more frequent and which territory is more vulnerable to insult. Finally, the correlations of the degree of saccular and PSCC dysfunction, as represented by the parameters of cVEMP and vHIT, were investigated to provide further insights into the extent of disease in the IVN.The results of this study can provide a basis for understanding vestibular pathologies and for further use of cVEMP and vHIT.

\section{MATERIALS AND METHODS}

\section{Participants}

We retrospectively reviewed patients who attended the dizziness clinic of Severance Hospital between July 2015 and December 2018. Inclusion criteria were (1) patients who were older than 19 years and (2) patients who were assessed using caloric tests, cVEMP, and vHIT on the same day. Patients older than 65 years who showed bilateral absent responses in cVEMP were excluded because bilateral cVEMP (no response) was reported to be common in older people, even in those with intact saccular and IVN function [6]. Since middle ear pathology could result in an abnormal VEMP response, we also excluded patients with middle ear disease. This study was approved by the Institutional Review Board of Severance Hospital (IRB No. 4-2018-1129). Owing to the retrospective design, the requirement for informed consent was waived.

\section{Classification of diseases}

Diseases were diagnosed and classified as follows: benign paroxysmal positional vertigo (BPPV); probable BPPV spontaneously resolved; Meniere disease; acute vestibular neuritis; chronic peripheral vestibulopathy; vestibular migraine; persistent posturalperceptual dizziness (PPPD); dizziness due to cardiogenic causes such as orthostatic dizziness or presyncope, recurrent vestibulopathy, central vertigo, bilateral vestibulopathy, physiologic dizziness, such as motion sickness, and fistula-induced dizziness, such as perilymphatic fistula or superior canal dehiscence syndrome. Diagnostic criteria for diseases used in the present study is provided as a supplementary file (Supplementary Material 1).

\section{Bithermal caloric test}

The bithermal caloric test was performed at water irrigation temperatures of $30^{\circ} \mathrm{C}$ and $44^{\circ} \mathrm{C}$, and the canal paresis (CP) value 
was calculated using Jongkees' formula [7]. We defined $>25 \%$ of Jongkee's index in the caloric test as evidence of impaired unilateral SVN function. Bilateral caloric weakness was defined as a total caloric response of $<12 \%$ sec according to the Bárány Society Consensus [8].

\section{Cervical vestibular evoked myogenic potential}

For the cVEMP test (Eclipse; Interacoustics, Middelfart, Denmark), active electrodes were placed on the upper half of the bilateral sternocleidomastoid muscles, while reference and ground electrodes were placed on the suprasternal notch and forehead, respectively. Electromyographic signals were amplified, bandpassfiltered between 30 and 3,000 $\mathrm{Hz}$, and monitored to maintain background muscle activity at over $50 \mu$ V.To obtain the cVEMP amplitude, acoustic stimuli were delivered through an insert earphone. Acoustic stimuli were $500 \mathrm{~Hz} 95 \mathrm{~dB}$ nHL short-tone bursts with rarefaction polarity (rise/fall time $1 \mathrm{~ms}$ each; plateau time $2 \mathrm{~ms}$; stimulation rate 5.10/sec). An average of 100 responses were recorded for each run, with the subject sitting with his/her head rotated sideways toward one shoulder to activate the sternocleidomastoid muscle [9]. The cVEMP was measured using monaural acoustic stimulation with ipsilateral recordings. The first positive and second negative polarities of the biphasic waveform were termed waves p13 and n23, respectively. Consecutive trials were performed to confirm the reproducibility of peaks p13 and n23, following which cVEMP responses were considered to be present. Conversely, cVEMP responses were considered absent when the biphasic p13-n23 waveform was not reproducible. IAD was calculated by comparing the rectified amplitude between the two sides since the amplitude could be greatly changed by age, auditory acuity, and muscle tone. In particular, it is directly affected by the contractile force of both cervical muscles, which increases the cervical muscle tone and shows greater amplitudes. Thus, the amplitude was rectified to correct the asymmetric muscle tones by dividing the raw amplitude generated by the acoustic stimulus by estimating the muscle contraction. IAD was calculated according to the formula: $\mathrm{IAD}=\left(\mathrm{A}_{\text {right }}-\mathrm{A}_{\text {left }}\right) /\left(\mathrm{A}_{\text {right }}+\mathrm{A}_{\text {left }}\right) \times 100$. The normal range of the IAD values from the authors' balance lab is summarized in Fig. 1. Considering that $95.4 \%$ of the normal IAD values were within the range of two standard deviations, which were 0 to $49.4 \%$, IAD $\geq 50 \%$ was considered pathologic. This cut-off value was the highest that was adopted in several studies [10].

\section{Video head impulse test}

A vHIT device from GN Otometrics (ICS Impulse; GN Otometrics, Taastrup, Denmark) was used to record eye movements. Default software settings were used. To evaluate the PSCC, participants were seated to face the target on the wall at a distance of $1 \mathrm{~m}$. With their head turned approximately $40^{\circ}$ to the right for testing in the left posterior plane or $40^{\circ}$ to the left for testing in the right posterior plane, the head impulses were conducted

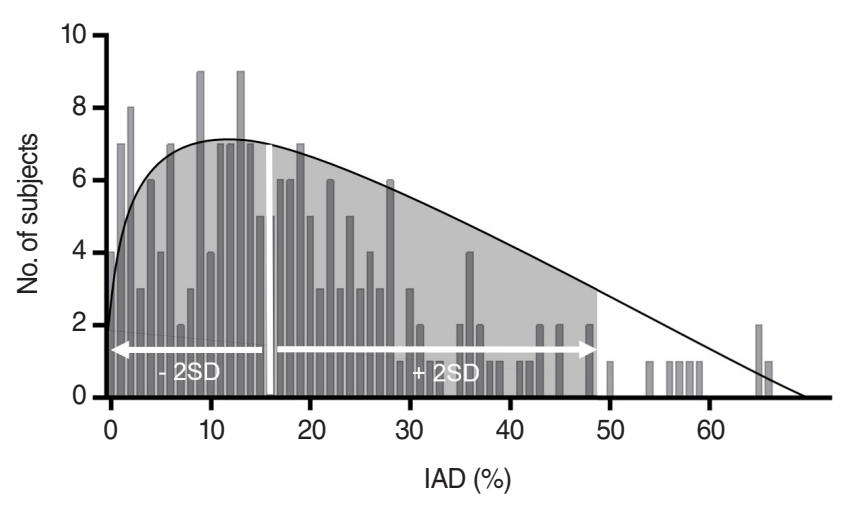

Fig. 1. Normal interaural amplitude difference (IAD) values calculated from 189 healthy subjects. The subjects were aged between 15 and 88 years (mean \pm standard deviation [SD], $41.1 \pm 18.4$ years), and 101 were men. The average IAD value was $19.9 \% \pm 14.7 \%$, and the reference range was $0 \%-49.3 \%$. According to the reference range, an $I A D \geq 50 \%$ was considered pathologic in the present study.

by the same right-handed examiner with a peak velocity range of $200^{\circ}-250^{\circ}$ per second, rotation amplitude of approximately $15^{\circ}$, and duration of $150-200 \mathrm{~ms}$. A minimum of 20 vertical head impulses were delivered randomly in the upward or downward direction for the evaluation of vertical canals, and the vestibulo-ocular reflex gain of the posterior semicircular canal (pVOR) were adopted for the analysis. The mean gain value for each side of the PSCC was calculated automatically. A mean gain value in the PSCC of less than 0.75 , was defined as the pathologic value [4].

\section{Statistical analysis}

Statistical analyses were conducted using the IBM SPSS ver. 22 (IBM Corp., Armonk, NY, USA). Descriptive statistics were examined for the data. Normality tests were performed on the vestibular function test parameters (CP, IAD, and p-VOR). The visual examination of Q-Q normality plots and the Shapiro-Wilk test suggested that all parameters satisfied normality. Hence, Pearson correlation analysis, which is a parametric test, was performed to examine the correlation between IAD and p-VOR. Chi-square tests were performed to examine the association between IVN involvement and SVN involvement. Statistical significance was set at $P<0.05$.

\section{RESULTS}

\section{Patient information and disease prevalence}

In total, 414 patients were enrolled in this study. The mean age of the enrolled subjects was $53.1 \pm 16.5$ years (range, $20-83$ years), and 301 were female. The final diagnoses of the patients were probable BPPV that spontaneously resolved $(n=131,31.6 \%)$, 
Table 1. Detected frequency of canal weakness, pathologic IAD in cVEMP, and pathologic p-VOR in VHIT for each diagnosis

\begin{tabular}{|c|c|c|c|c|}
\hline Variable & Patient & Canal weakness & Pathologic IAD ratio & Pathologic p-VOR \\
\hline Probable BPPV, spontaneously resolved & $131(31.6)$ & $33(25.2)$ & $44(33.6)$ & $20(15.3)$ \\
\hline Meniere disease & $56(13.5)$ & $21(37.5)$ & $23(41.1)$ & $11(19.6)$ \\
\hline Acute vestibular neuritis & $47(11.4)$ & $29(61.7)$ & $17(36.2)$ & $7(14.9)$ \\
\hline Chronic peripheral vestibulopathy & $39(9.4)$ & $11(28.2)$ & $20(51.3)$ & $5(12.8)$ \\
\hline Vestibular migraine & $37(8.9)$ & $5(13.5)$ & $12(32.4)$ & $3(8.1)$ \\
\hline PPPD & $30(7.2)$ & $9(30.0)$ & $11(36.7)$ & $3(10.0)$ \\
\hline Cardiogenic causes & $28(6.8)$ & $5(17.9)$ & $11(39.3)$ & $2(7.1)$ \\
\hline BPPV & $14(3.4)$ & $3(21.4)$ & $5(35.7)$ & $1(7.1)$ \\
\hline Recurrent vestibulopathy & $11(2.7)$ & $1(9.1)$ & $3(27.3)$ & 0 \\
\hline Central vertigo & $7(1.7)$ & $1(14.3)$ & $1(14.3)$ & $2(28.6)$ \\
\hline Bilateral vestibulopathy & $6(1.4)$ & 0 & $3(50.0)$ & $3(50.0)$ \\
\hline Physiologic dizziness & $4(1.0)$ & 0 & $1(25.0)$ & $1(25.0)$ \\
\hline Fistula-induced dizziness & $4(1.0)$ & $1(25)$ & $1(25.0)$ & 0 \\
\hline
\end{tabular}

Values are presented as number (\%).

IAD, interaural amplitude difference; cVEMP, cervical vestibular evoked myogenic potential; $p$-VOR, vestibulo-ocular reflex gain of the posterior semicircular canal; vHIT, video head impulse test; BPPV, benign paroxysmal positional vertigo; PPPD, persistent postural-perceptual dizziness.

Meniere disease $(n=56,13.5 \%)$, acute vestibular neuritis $(n=47$, $11.4 \%)$, chronic peripheral vestibulopathy $(n=39,9.4 \%)$, and vestibular migraine $(n=37,8.9 \%)$. PPPD $(n=29,7.2 \%)$, dizziness due to cardiogenic causes such as orthostatic dizziness or presyncope $(n=28,6.8 \%)$, BPPV $(n=14,3.4 \%)$, recurrent vestibulopathy ( $\mathrm{n}=11,2.7 \%)$, central vertigo $(\mathrm{n}=7,1.7 \%)$, and bilateral vestibulopathy $(n=6,1.4 \%)$. Physiologic dizziness, such as motion sickness $(n=4,1.0 \%)$, perilymphatic fistula, or superior canal dehiscence syndrome $(n=4,1.0 \%)$, were also reported. Table 1 shows the detected frequency of canal weakness, pathologic IAD in cVEMP, and pathologic p-VOR in vHIT for each diagnosis. Patients with PPPD, cardiogenic dizziness, central vertigo, and physiologic dizziness were excluded from further analysis because the pathophysiology of these diseases is limited to the vestibular system; thus, a final total of 346 patients were included in the following analysis.

\section{Extent of vestibular pathology in the enrolled patients}

First, we analyzed the extent of vestibular pathology using the caloric test, vHIT, and cVEMP from 346 patients. In total, 104 patients $(30.1 \%)$ showed unilateral or bilateral caloric weakness, and 156 patients (45.1\%) showed either pathologic IAD and/or p-VOR values. Among them, 54 patients (15.6\%) showed both caloric weakness and pathologic IAD and/or p-VOR values. Since caloric weakness represents impaired SVN function, and pathologic IAD and/or p-VOR values represent impaired IVN function, a total of 54 patients $(15.6 \%)$ had total VN dysfunction, 50 (14.5\%) had solely SVN dysfunction, and 102 (29.5\%) had solely IVN dysfunction. Abnormal results were more frequently found in vestibular tests investigating the IVN than in vestibular tests investigating the SVN in patients with general vestibular disorders.

Next, we categorized the subjects into four subgroups to in-
Table 2. Prevalence of pathologic IAD ratios and pathologic p-VOR

\begin{tabular}{lccr}
\hline \multirow{2}{*}{ Variable } & \multicolumn{2}{c}{ IAD ratio } & \\
\cline { 2 - 3 } & $\begin{array}{c}\text { Within normal } \\
\text { range (n) }\end{array}$ & Pathologic (n) & \\
\hline$p$-VOR & & & \\
Within normal range (n) & Group 1: 190 & Group 2: 106 & 296 \\
Pathologic (n) & Group 3: 27 & Group 4: 23 & 50 \\
Total $(n)$ & 217 & 129 & 346 \\
\hline
\end{tabular}

IAD, interaural amplitude difference; p-VOR, vestibulo-ocular reflex gain of the posterior semicircular canal.

vestigate the precise pathological location in the IVN territory, as follows: group 1, patients with normal IAD and normal p-VOR; group 2, patients with pathologic IAD and normal p-VOR; group 3, patients with normal IAD and pathologic p-VOR; and group 4, patients with both pathologic IAD and p-VOR.Among the total enrolled patients $(n=346), 190$ were in group 1, 106 in group 2, 27 in group 3, and 23 in group 4 (Table 2). The distribution of patients with abnormal caloric test results in each group was as follows: 50 of $190(26.3 \%)$ in group 1, 34 of $106(32.1 \%)$ in group 2, 9 of $27(33.3 \%)$ in group 3, and 11 of $23(47.8 \%)$ in group 4 . There was no significant difference in the proportion of patients with pathologic caloric test results among the groups $(P>0.05$, chi-square test).

\section{The relationship between IAD in cVEMP and p-VOR in vHIT} We further analyzed the relationship between the IAD in cVEMP and p-VOR in vHIT by performing a Pearson correlation analysis in 346 patients. Since IAD was obtained by subtracting the left-sided amplitude from the right-sided amplitude, a positive IAD represented the presence of a pathology on the left side. Thus, we matched positive IAD values with $[1-(\mathrm{p}-\mathrm{VOR}$ in left 

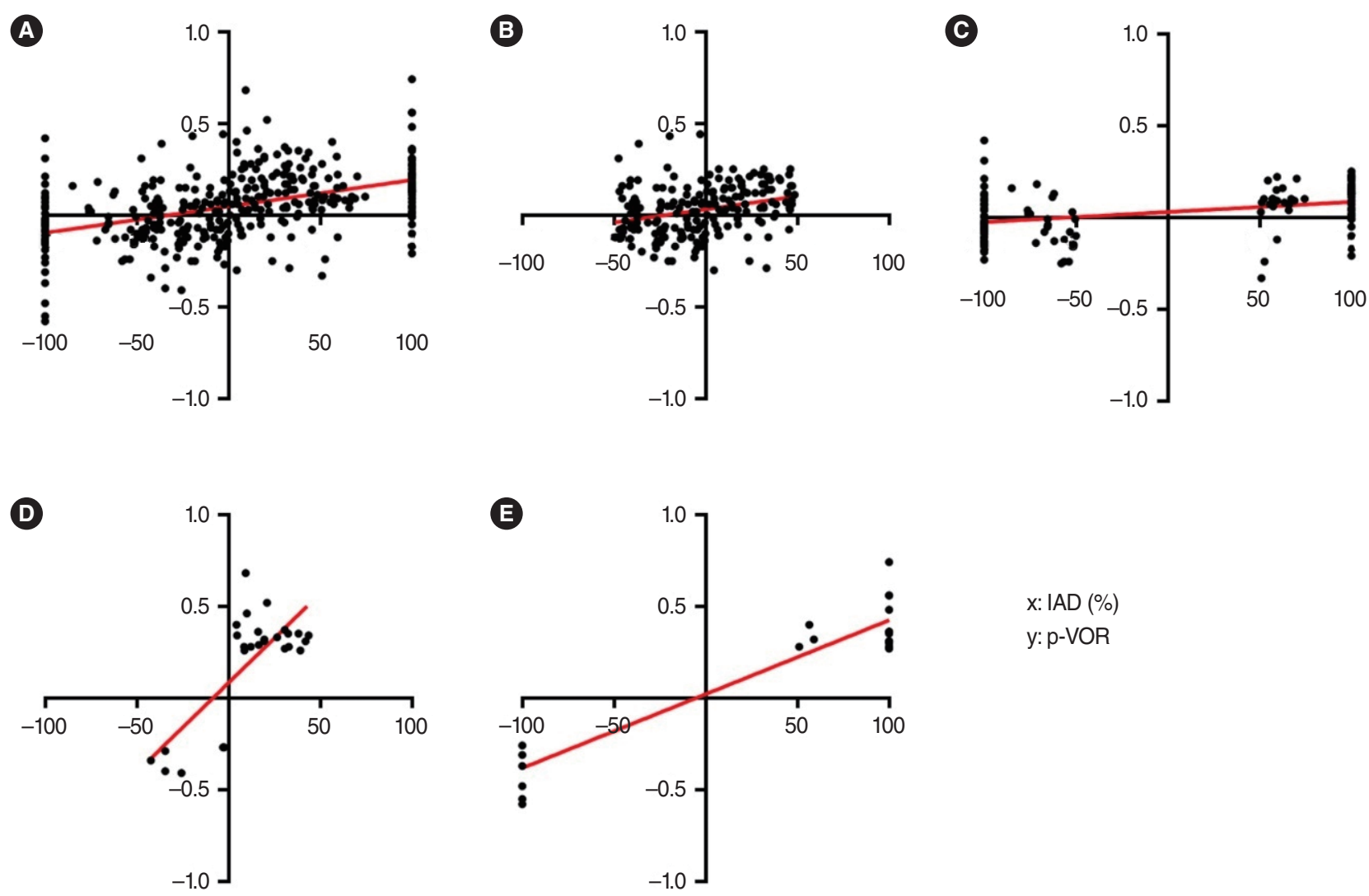

$$
\begin{aligned}
& x: \operatorname{IAD}(\%) \\
& y: p-V O R
\end{aligned}
$$

Fig. 2. The correlation between the interaural amplitude difference (IAD) and the vestibulo-ocular reflex gain of the posterior semicircular canal ( $p$-VOR). They were significantly correlated in the total population ( $\mathrm{A}: \mathrm{n}=346, r=0.440, P<0.01)$. The correlations were also statistically significant in four subgroups: (1) patients who had normal IAD and normal p-VOR (B: $n=190, r=0.284, P<0.01)$, (2) patients who had pathologic IAD and normal p-VOR (C: $\mathrm{n}=106, r=0.339, P<0.01)$, (3) patients who had normal IAD and pathologic $p-V O R(D: n=27, r=0.762, P<0.01)$, and (4) patients with both pathologic IAD and pathologic $p-\operatorname{VOR}(E: n=23, r=0.944, P<0.01)$.

PSCC)] and negative IAD values with -[1-(p-VOR in right PSCC)] to analyze the correlation between IAD and PSCC gain. IAD was significantly correlated with $\mathrm{p}-\mathrm{VOR}$ in the total population $(r=0.440, P<0.01)$ (Fig. 2A). We examined the correlation between IAD and $\mathrm{p}-\mathrm{VOR}$ in each group, as described above. The analysis revealed that both tests had significant correlations in all subgroups with different $r$-values. The order of the strength of correlation between the two tests was group $1(r=0.284, P<0.01)$ $<$ group $2(r=0.339, P<0.01)<$ group $3(r=0.762, P<0.01)$ $<$ group 4 ( $r=0.944, P<0.01)$ (Fig. 2B-E).

\section{DISCUSSION}

To the best of our knowledge, this is the first report to investigate the correlations between two inferior vestibular function testsnamely, the cVEMP and vHIT for PSCC - in a large population with a variety of general vestibular disorders. The main findings of this study are summarized as follows: First, abnormal results were more frequently found in vestibular tests investigating the IVN than in vestibular tests investigating the SVN in patients with general vestibular disorders. Second, isolated saccular or PSCC pathology is likely to be more common than combined pathology in patients with IVN dysfunction. In particular, isolated saccular dysfunction seems to be more common in these patients, which means that the saccule is more vulnerable to insult. However, this finding could be elicited from different sensitivities according to the normal values of cVEMP and vHIT, which will be discussed below. Third, pathologic p-VOR was highly likely to be accompanied by saccular pathology; however, the IAD value alone could not predict the combined dysfunction of the saccule and PSCC because isolated saccular dysfunction was more common.

The IVN is composed of the saccular and posterior ampullary nerves, each of which innervates the saccule and PSCC. cVEMP assesses the function of the saccule and saccular nerves, and vHIT for the PSCC assesses the function of the posterior canal ampulla and posterior ampullary nerve. These two tests examine the different branches of the IVN and the corresponding end organs. Thus, the results of cVEMP and p-VOR were closely correlated in patients with proximal IVN dysfunction. Indeed, several studies have reported a substantial correlation between the re- 
sults of the two tests. Park et al. [3] studied the inter-test agreement between cVEMP and vHIT for the PSCC in patients with vestibular neuritis, and fair agreement between the two tests was observed. Similarly, oVEMP for the utricle and vHIT for the lateral semicircular canal, both of which are innervated by the SVN, were compared in another study. The two tests also showed a significant correlation in patients with vestibular neuritis [4]. Walther and Blodow [5] assessed patients with vestibular neuritis using cVEMP, oVEMP, and vHIT and classified them into several subtypes according to the test results. These studies suggest that the proximal portion of each IVN and SVN is more likely to be affected by vestibular neuritis, rather than having an isolated end organ pathology. However, these studies were conducted in patients with acute vestibular neuritis, and studies investigating the extent of the pathology in the vestibular organs in patients with general vestibular disorders are insufficient. Therefore, we extended the study to subjects who had not only acute vestibulopathy but also chronic or recurrent dizziness/vertigo and investigated the correlation between the two tests in a larger population. We found that the IVN territory was likely to be more vulnerable to injury than the SVN territory, as suggested by the higher number of patients with either pathologic cVEMP and/or p-VOR gain in vHIT than the number of patients with pathologic caloric test results. In addition, the prevalence of saccular dysfunction $(82.0 \%)$ was greater than that of PSCC dysfunction (32.6\%). It is assumed that the saccular nerve is more vulnerable than the posterior ampullary nerve, which is in accordance with a previous report [3]. Gianoli et al. [11] explained different vulnerabilities among different divisions of the vestibular nerve based on anatomical differences. Each nerve passes through bony canals and has different available spaces. The IVN innervating the saccule had less room for swelling than the posterior ampullary nerve. Thus, nerve entrapment is more frequent in the saccular nerve than in the posterior ampullary nerve in cases of vestibular neuritis or other IVN vestibular pathologies.

The aforementioned findings could have resulted from the different sensitivities of each test tool. Additionally, the issue of specificity cannot be overlooked. The findings of the present study were based on a test focusing on sensitivity, but specificity is often regarded as more important in this kind of study. The Pearson correlation analyses between cVEMP and p-VOR showed close correlations in the four groups, even in the normal population. This means that we could miss the "real" pathology in the saccule and posterior canal ampulla, or their innervating nerves, with the borderline value being close to the normal limit; this problem could result in a specificity issue. In addition, different end organ cell types between the saccule and the posterior ampullary crest might have caused the difference in the results between cVEMP and vHIT. The differences in abnormalities between cVEMP and vHIT may reflect one afferent cell type being more sensitive to pathology than the other. Despite several limitations of each test, the real sensitivity of each test is difficult to find because the real pathological extent and human cellular sensitivity cannot be identified with other laboratory or imaging tools currently available in clinical settings. Studies have reported varying sensitivities of cVEMP and the caloric test; however, there is a limitation that no definite standard exists for establishing the sensitivity of each test $[12,13]$. Therefore, we deduced the results of a higher prevalence of IVN and saccular pathology based on the current evaluation tools, although the results might be imperfect. Another possibility to explain the higher prevalence of IVN and saccular pathology might be the higher proportion of vestibular disorders. In this study population, BPPV, including probable BPPV that spontaneously resolved, accounted for the highest proportion of cases. Otolithic dysfunction detected in the VEMP test is more frequently found in patients with BPPV than in the normal population [14]. However, pathologic VEMP findings in patients with BPPV did not significantly affect the overall outcome $(P=0.464)$, and the prevalence of IVN and saccular pathology was likely to be higher than that of SVN pathology among patients with general vestibular disorders in this study.

We analyzed the correlation between the two tests using IAD and $\mathrm{p}$-VOR in the four subgroups. The correlation was most significant in patients with both pathologic IAD and pathologic $\mathrm{p}$ VOR, with an $r$-value of 0.947 . Patients with both normal IAD and normal p-VOR showed a statistically significant correlation, but this correlation was the weakest $(r=0.297)$. This result may indicate that even in patients with normal-range values in both tests, mildly decreased proximal IVN function can be present in certain proportions of the population. Interestingly, the correlation was stronger in patients with normal IAD and pathologic p-VOR $(r=0.768)$ than in patients with pathologic IAD and normal p-VOR $(r=0.382)$. It is strongly suspected that if patients presented with a pathologic p-VOR gain in vHIT, they would have a common pathology for both the saccule and the PSCC, or the proximal IVN. Thus, for patients who showed a pathologic p-VOR gain in vHIT and a normal IAD at first, it was tempting to speculate that this finding indicated borderline injury and that the IAD could progress to a pathologic value in the near future. In contrast, patients with pathologic IAD and normal p-VOR showed a weaker correlation between the two tests, suggesting that isolated saccular dysfunction would be more common in this group. Taken together, the four subgroups resulting from the combination of two test outcomes are suspected of having different clinical pathways, such as the degree of symptoms or disease progression. Thus, it is recommended to perform these two tests simultaneously when evaluating subjects in the clinical setting since combining the results of the two tests could elucidate the patient's clinical pathway. However, this should be investigated in a well-designed prospective study to provide more concrete evidence.

We found that abnormal results were more frequent in vestib- 
ular tests investigating the IVN than in vestibular tests investigating the SVN in patients with general vestibular disorders, which might be interpreted as indicating that pathology is more commonly present in the IVN territory than in the SVN territory in patients with general vestibular disorders. In particular, based on the results of the current study, it is tempting to speculate that isolated saccular and PSCC pathologies are more common and that saccular pathology is likely to be predominant in the inner ear territory innervated by the IVN. In addition, patients with pathologic p-VOR gain in vHIT had a higher probability of having both saccular and PSCC pathologies than patients with pathologic IAD results. The results of this study suggest the importance of considering the presence of pathology in the IVN territory in the evaluation of patients with vestibular disorders. We believe that this study also provides basic information on the use of cVEMP and vHIT in the evaluation of patients with general vestibular disorders, especially in those with IVN pathology.

\section{CONFLICT OF INTEREST}

No potential conflict of interest relevant to this article was reported.

\section{ORCID}

Jeon Mi Lee https://orcid.org/0000-0003-2793-9045

Hyun Jin Lee $\quad$ https://orcid.org/0000-0003-3826-8830

Jungghi Kim https://orcid.org/0000-0002-6591-1732

Seung Ho Shin https://orcid.org/0000-0001-9352-8426

Gina Na https://orcid.org/0000-0001-8111-2124

Dae Bo Shim https://orcid.org/0000-0002-2331-5000

Sung Huhn Kim https://orcid.org/0000-0003-4408-3066

\section{AUTHOR CONTRIBUTIONS}

Conceptualization: SHK. Data curation: JK, SHS, GN, DBS. Methodology: HJL. Formal analysis: HJL. Writing-original draft: JML.Writing-review and editing: JML, SHK.

\section{SUPPLEMENTARY MATERIALS}

Supplementary materials can be found online at https://doi.org/ 10.21053/ceo.2021.00794.

\section{REFERENCES}

1. Schubert MC, Minor LB. Vestibulo-ocular physiology underlying vestibular hypofunction. Phys Ther. 2004 Apr;84(4):373-85.

2. Taylor RL, McGarvie LA, Reid N, Young AS, Halmagyi GM, Welgampola MS. Vestibular neuritis affects both superior and inferior vestibular nerves. Neurology. 2016 Oct;87(16):1704-12.

3. Park JS, Kim CH, Kim MB. Comparison of video head impulse test in the posterior semicircular canal plane and cervical vestibular evoked myogenic potential in patients with vestibular neuritis. Otol Neurotol. 2018 Apr;39(4):e263-8.

4. Skoric MK, Adamec I, Pavicic T, Pavlovic I, Ruska B, Crnosija L, et al. Vestibular evoked myogenic potentials and video head impulse test in patients with vertigo, dizziness and imbalance. J Clin Neurosci. 2017 May;39:216-20.

5. Walther LE, Blodow A. Ocular vestibular evoked myogenic potential to air conducted sound stimulation and video head impulse test in acute vestibular neuritis. Otol Neurotol. 2013 Aug;34(6):1084-9.

6. Piker EG, Jacobson GP, Burkard RF, McCaslin DL, Hood LJ. Effects of age on the tuning of the cVEMP and oVEMP. Ear Hear. 2013 Nov-Dec;34(6):e65-73.

7. Jongkees LB, Maas JP, Philipszoon AJ. Clinical nystagmography: a detailed study of electro-nystagmography in 341 patients with vertigo. Pract Otorhinolaryngol (Basel). 1962;24:65-93.

8. Strupp M, Kim JS, Murofushi T, Straumann D, Jen JC, Rosengren SM, et al. Bilateral vestibulopathy: Diagnostic criteria Consensus document of the Classification Committee of the Barany Society. J Vestib Res. 2017 Oct;27(4):177-89.

9. Chou CH,Wang SJ,Young YH. Feasibility of the simultaneous ocular and cervical vestibular-evoked myogenic potentials in unilateral vestibular hypofunction. Clin Neurophysiol. 2009 Sep;120(9):1699705 .

10. Blakley BW, Wong V. Normal values for cervical vestibular-evoked myogenic potentials. Otol Neurotol. 2015 Jul;36(6):1069-73.

11. Gianoli G, Goebel J, Mowry S, Poomipannit P. Anatomic differences in the lateral vestibular nerve channels and their implications in vestibular neuritis. Otol Neurotol. 2005 May;26(3):489-94.

12. Verbecque E, MarijnissenT, De Belder N,Van RompaeyV, Boudewyns A, Van de Heyning P, et al. Vestibular (dys)function in children with sensorineural hearing loss: a systematic review. Int J Audiol. 2017 Jun;56(6):361-81.

13. Zapala DA, Brey RH. Clinical experience with the vestibular evoked myogenic potential. J Am Acad Audiol. 2004 Mar;15(3):198-215.

14. Xu H, Liang FY, Chen L, Song XC, Tong MC, Thong JF, et al. Evaluation of the utricular and saccular function using oVEMPs and cVEMPs in BPPV patients. J Otolaryngol Head Neck Surg. 2016 Feb;45:12. 